\title{
Toxina Botulínica como tratamiento de la espasticidad en la parálisis cerebral infantil.
}

\author{
Caso Clínico
}

Alejandro Ojeda-Manzano.

Facultad de Medicina. Licenciatura en Rehabilitación, Universidad Autónoma de Yucatán, Mérida, Yucatán, México.

\section{RESUMEN.}

Introducción. Uno de los factores que contribuyen a la discapacidad del niño con parálisis cerebral infantil (PCI) es la presencia de espasticidad como limitante de la función motora. La toxina botulínica tipo A (TBA) inyectada dentro de la musculatura espástica, es una nueva modalidad terapéutica en este tipo de pacientes.

Caso clínico. Paciente del sexo femenino de 6 años de edad con diagnóstico de PCI ocasionada por hipoxia neonatal, con tetraparesia espástica. Se inyectó TBA en los músculos seleccionados por la exploración clínica. Se consideró una respuesta positiva al tratamiento: la disminución de la espasticidad según la escala modificada de Ashworth y una puntuación igual o superior a tres en la escala de mejoría funcional, calificada por los padres, terapeuta y médico (escala global de O’Brien). El tratamiento con TBA estuvo acompañado en todo momento de terapia física. A los dos meses de la inyección con toxina botulínica se obtuvieron los siguientes resultados: según la escala de Ashworth de un grado 4 disminuyó a un grado 3 en los músculos isquiotibiales y flexor corto del pulgar; y de un grado 4 a un grado 2, en la misma escala, en los músculos flexor superficial de dedos, bíceps braquial y braquiradial; y de acuerdo a escala de O’Brien se consideró en un grado 5.

Discusión. Los buenos resultados del uso de la TBA, acompañada de una adecuada terapia física, en los problemas de espasticidad en el paciente con PCI son confirmados en este caso. La intensidad del efecto de la TBA depende de la espasticidad de cada caso, tratamiento previo, sensibilidad del paciente a TBA, técnica de aplicación y la terapia física posterior.

(Rev Biomed 2004; 15:107-112)

Palabras clave: Toxina botulínica, espasticidad, parálisis cerebral infantil.

\section{SUMMARY.}

Treatment of the spasticity in the infantile cerebral palsy with botulinum toxin.

Introduction. One of the factors that contributes to

Solicitud de sobretiros: Lic. en R. Alejandro Ojeda-Manzano, Calle 33 No. 110 x 74 y 76, C.P. 97320, Progreso, Yucatán, México. Correo electrónico: alex@multired.net.mx

Recibido el 14/Noviembre/2003. Aceptado para publicación el 20/Febrero/2004.

Este artículo está disponible en http://www.uady.mx/sitios/biomedic/revbiomed/pdf/rb041525.pdf 


\section{A Ojeda-Manzano.}

the disability of a child with infantile cerebral palsy (ICP) it is the presence of spasticity as a restrictive factor of the function of motor muscles. Botulinum Toxin type A (BTA) injected inside the spastic musculature, is a new therapeutic modality in this type of patient.

Clinical case. A 6 year-old female patient with a background of ICP caused by neonatal asphyxia and with a clinical record of spastic tetraparesis was examined. BTA was injected into the muscles selected by clinical exploration. A response was considered positive to the treatment: on the decrease in the degree according to Ashworth`s scale and the same score or superior at three in the scale of global improvement of O’Brien. The treatment with BTA was accompanied at all times by physical therapy. After two months of injection with BTA the following results were obtained: According to the scale of Ashworth a grade 4 diminished to a grade 3 in the biceps cruris, semitendinosus, semimembranosus, and flexor pollicis brevis muscles; and a grade 4 to a grade 2 in the same scale in the flexor digitorum sublimiss, biceps braquialis and braquiradialis muscles; and according to $\mathrm{O}$ Brien`s scale it was considered a grade 5 .

Discussion. The good results of the use of BTA accompanied by appropriate physical therapy in the spasticity problems in the patient with ICP have once again been proved in this case. The intensity of the effect of BTA depends on the spasticity in each different case, previous treatment, patient's sensitivity to BTA, technical application and physical therapy after the application. (Rev Biomed 2004; 15:107-112)

Key words: Botulinum toxin, spasticity, infantile cerebral palsy.

\section{INTRODUCCIÓN.}

Uno de los factores que contribuyen a la discapacidad del niño con parálisis cerebral infantil (PCI), es la presencia de espasticidad como factor limitante de la función motora. En los últimos años se han propuesto múltiples posibilidades terapéuticas, ninguna definitiva, con la finalidad de mejorar este síntoma. La toxina botulínica A (TBA) tiene diversas

\section{Revista Biomédica}

aplicaciones técnicas; inyectada dentro de la musculatura espástica es una nueva modalidad terapéutica en este tipo de pacientes (1-3).

La TBA pertenece a una familia de proteínas, producidas por la bacteria Clostridium botulinum, con capacidad para provocar una debilidad muscular o una parálisis parcial (denervación química). Su mecanismo de acción radica en la inhibición de la liberación de acetilcolina en la unión neuromuscular al unirse a las terminaciones nerviosas presinápticas, evitando la despolarización normal $(4,6)$.

La respuesta clínica depende de factores propios de la espasticidad, técnica de aplicación, sensibilidad del paciente a TBA (6). En general su efectividad dura de dos a seis meses $(7,8)$ y su precio es muy elevado. En Yucatán, México, oscila alrededor de los $\$ 5000$ pesos (460 dólares americanos). Los niños con espasticidad secundaria a PCI responden mejor si el tratamiento se aplica entre los dos y seis años de edad (1). La TBA es una herramienta más para tratar la espasticidad, muy fácil de utilizar, bastante efectiva y segura si se administra correctamente $(9,10)$.

\section{REPORTE DE CASO.}

Se ha realizado el seguimiento de un paciente de 6 años de edad, con antecedente de hipoxia neonatal, con cuadro clínico de tetraparesia espástica secundaria a PCI. Con un desarrollo motor en 5 meses, lenguaje adecuado para su edad y nivel intelectual conservado, con antecedente quirúrgico de tenotomía bilateral en tendón de Aquiles. La evaluación de la espasticidad

\section{Cuadro 1}

Valoración del tono muscular. Escala modificada de Ashworth (6).

\footnotetext{
$0 \quad$ Ningún aumento del tono.

1 Aumento discreto del tono con resistencia mínima al movimiento pasivo.

1+ Aumento discreto del tono con resistencia en todo el movimiento pasivo.

2 Aumento marcado del tono pero con movimiento pasivo fácil.

3 Aumento marcado del tono con dificultad para el movimiento pasivo.

$4 \quad$ Rigidez espástica severa en flexión o en extensión.
} 
Tratamiento de la espasticidad con toxina botulínica.

\section{Cuadro 2 \\ Escala modificada de mejoría global. Escala de O’Brien (4).}

1. Empeoramiento leve.

2. Sin cambios.

3. Mejoría leve.

4. Mejoría moderada sin cambios en la función.

5. Mejoría moderada de la función.

6. Mejoría marcada del tono y de la función.

se realizó con la escala de Ashworth (cuadro 1) y la escala de mejoría global de O’Brien, que consiste en una evaluación subjetiva de la mejoría, valorada por padres, terapeuta y médico (cuadro 2).

La exploración física de la paciente mostró un peso de $20 \mathrm{Kg}$ y se encontró con hipertonía, en un grado 4 según la escala de Ashworth, hiperreflexia y sensibilidad conservada en miembros superiores e inferiores, acompañada de contracturas dinámicas en isquiotibiales. En cuanto a la función en miembros superiores, la paciente podía dirigir intencionadamente la dirección de sus brazos, pero no lograba la

Cuadro 3

Dosis recomendadas en la infancia (10).

\begin{tabular}{lll}
\hline MÚSCULO & $\begin{array}{c}\text { U/Kg de peso } \\
\text { corporal. } \\
\text { Toxina Botulínica }\end{array}$ & $\begin{array}{c}\text { Puntos de } \\
\text { inyección }\end{array}$ \\
\hline Triceps Sural & $3-6$ & $2-4$ \\
Tibial posterior & $1-2$ & 1 \\
Adductores del Muslo & $2-3$ & 2 \\
Isquiotibiales & $2-3$ & $2-3$ \\
Bíceps braquial & 3 & 2 \\
Braquiorradial & $2-3$ & $1-2$ \\
Deltoides & $2-3$ & 3 \\
Flexores del carpo & & \\
(dosis a repartir entre & & $2-4$ \\
los músculos) & $2-3$ & $1-2$ \\
Flexores de dedos & $1-2$ & $1-2$ \\
Pronador redondo & 1 & 1 \\
Eminencia tenar & $1-2$ & 1 \\
Otros músculos pequeños & $0.5-1$ & \\
NOTA: DOSIS MÁXIMA: & & \\
Total & $400 \mathrm{U}$ ó 12 U/Kg \\
Por músculo & $150 \mathrm{U}$ & \\
Por punto de inyección & $50 \mathrm{U}$ & \\
\hline
\end{tabular}

Cuadro 4

Técnica utilizada en el caso.

\begin{tabular}{llcc}
\hline Músculo & Dosis & $\begin{array}{c}\text { Cantidad de } \\
\text { Unidades } \\
\text { de TBA }\end{array}$ & $\begin{array}{c}\text { Puntos de } \\
\text { Inyección }\end{array}$ \\
\hline Bíceps Crural & $1.3 \mathrm{Ux} \mathrm{Kg}$. & $30 \mathrm{U}$ & 2 \\
Semimembranoso & $.5 \mathrm{Ux} \mathrm{Kg}$. & $10 \mathrm{U}$ & 1 \\
Semitendinoso & $.5 \mathrm{Ux} \mathrm{Kg}$. & $10 \mathrm{U}$ & 1 \\
Bíceps braquial & $1 \mathrm{Ux} \mathrm{Kg}$. & $20 \mathrm{U}$ & 1 \\
Braquiradial & $.4 \mathrm{Ux} \mathrm{Kg}$. & $8 \mathrm{U}$ & 1 \\
$\begin{array}{l}\text { Flexor superficial de } \\
\text { dedos }\end{array}$ & $.5 \mathrm{Ux} \mathrm{Kg}$. & $10 \mathrm{U}$ & 1 \\
Flexor corto del pulgar & $.25 \mathrm{Ux} \mathrm{Kg}$. & $5 \mathrm{U}$ & 1 \\
\hline
\end{tabular}

extensión de los dedos y la mano. El control de tronco lo lograba al permanecer sedente sin apoyo. En miembros inferiores la bipedestación no era alcanzada, y se consideró la contractura de los músculos isquiotibiales como causa principal de este problema. Con base en los hallazgos encontrados en la exploración física, se propone el tratamiento de la TBA con el objetivo de aumentar la funcionalidad en general de la paciente, disminuyendo el tono de los músculos inyectados.

Para la aplicación del tratamiento se utilizó el cálculo de unidades de TBA por kg de peso corporal de la paciente y el punto o número de puntos de inyección intramuscular, que deben ser aplicados lo más cercano posible a la placa neuromuscular de cada músculo para lograr el efecto de bloqueo en la liberación de acetilcolina. Se inyectó TBA a diferentes dosis (cuadros 3 y 4), debido a que para cada músculo, la dosis cambia; esto es por su tamaño y funcionalidad.

Estos músculos fueron valorados clínicamente, seleccionando los músculos con mayor responsabilidad en la función; para la extensión de los dedos y mano (agarrar-soltar) se escogieron flexor superficial de dedos y flexor corto del pulgar, y para el movimiento de la mano-comida-boca (alimentación) el bíceps braquial y braquirradial; buscando mejorar el control en general de los miembros superiores y con ello aumentar el control del tronco. En los miembros inferiores se seleccionaron los músculos 
A Ojeda-Manzano.

\section{Cuadro 5 \\ Comparación de la puntuación Ashworth antes y después del tratamiento.}

\begin{tabular}{lcc}
\hline Músculo & $\begin{array}{c}\text { Antes del } \\
\text { tratamiento }\end{array}$ & $\begin{array}{c}\text { Después } \\
\text { del tratamiento }\end{array}$ \\
\hline Isquitibiales & 4 & 3 \\
Bíceps braquial & 4 & 2 \\
Braquirradial & 4 & 2 \\
Flexor superficial & & \\
de dedos & 4 & 2 \\
Flexor corto del pulgar & 4 & 3 \\
\hline
\end{tabular}

isquiotibiales (semimembranoso, semitendinoso y bíceps crural), que debido a su contractura dinámica imposibilitan la bipedestación y, por consiguiente, la marcha, siendo estas dos últimas las funciones a alcanzar con el tratamiento de TBA.

Se indicó, además del tratamiento con TBA, una terapia física diseñada específicamente para disminuir la espasticidad y potenciar el efecto de la TBA. Para este fin, se utilizaron las técnicas de neurodesarrollo y el método Kabat (diagonales en espiral), con el objetivo de aumentar el Arco de Movilidad Pasivo (AMP), mejorar la función y la coordinación tanto gruesa como fina, así como evitar la cirugía ortopédica correctora, en este caso la tenotomía isquiotibial.

Las evaluaciones se realizaron antes del

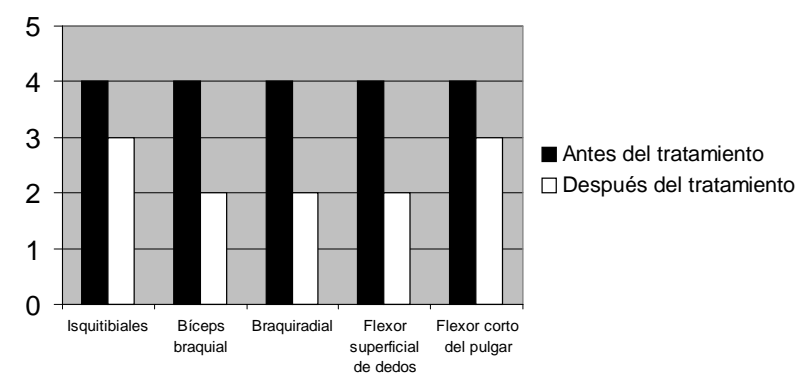

Figura 1.- Comparativa de músculos infiltrados, según la escala Ashworth, antes y después del tratamiento. Se obtuvieron mejores resultados en los músculos de los miembros superiores que en los miembros inferiores, a excepción del flexor corto del pulgar, que al igual que los isquiotibiales, disminuyó un grado en la escala, difiriendo de los demás músculos donde se obtuvo una mejoría en dos grados.

\section{Revista Biomédica}

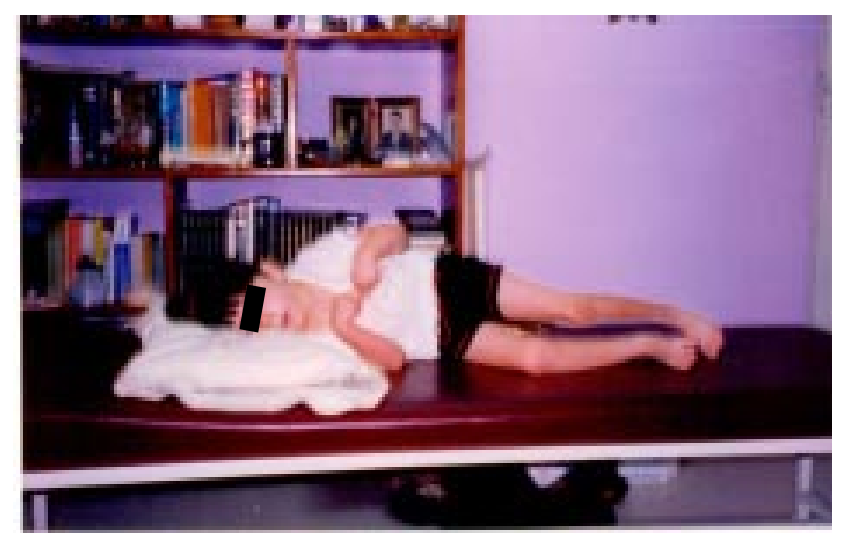

Figura 2.- Paciente antes del tratamiento. En la foto se observa el patrón flexor espástico en brazos, antebrazos, manos y dedos, limitante en la función de los miembros superiores (marzo 2003).

tratamiento y a los 2 meses de la inyección, donde se incluyeron las observaciones de los familiares (escala de O’Brien).

\section{RESULTADOS.}

Los resultados alcanzados después de dos meses de la aplicación de TBA fueron los siguientes: de un grado 4 (rigidez espástica severa en flexión o en extensión.) disminuyó a un grado 3 (aumento marcado del tono con dificultad para el movimiento pasivo), según la escala de Ashworth, en los músculos isquiotibiales y flexor corto del pulgar; de un grado 4 a un grado 2 (aumento marcado del tono, pero con movimiento pasivo fácil) los músculos flexor superficial de dedos, bíceps braquial y braquirradial (cuadro 5 y figura 1). De acuerdo a escala de O'Brien, se consideró en un grado 5 (mejoría moderada de la función). En cuanto a la función, la paciente logró la función manual de agarrar-soltar (figura 2), así como de alimentarse independientemente, se mejoró el control de tronco en dos puntos con apoyo (figura 3) y en miembros inferiores no se obtuvo mejora en la función. No se observó ningún efecto secundario y/o complicación alguna en la paciente.

\section{DISCUSIÓN.}

El resultado hacia la mejoría confirma los comunicados en la literatura, en cuanto a la espasticidad (1,2,4,6-9,11-16). Los beneficios 


\section{Tratamiento de la espasticidad con toxina botulínica.}

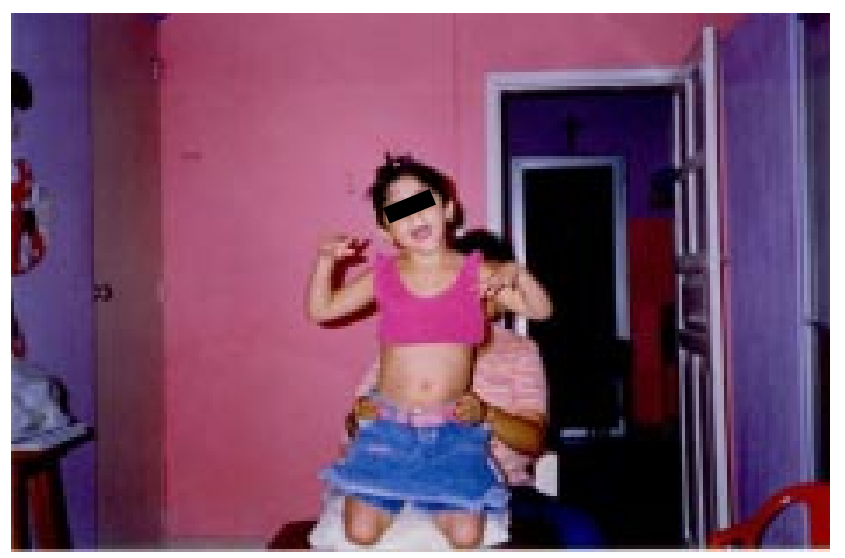

Figura 3.- Paciente después del tratamiento. En la foto se puede observar la mejoría en la función de la mano; el patrón flexor permanece. Sin embargo el tono muscular ha disminuido, aumentando la capacidad de extensión de antebrazos, muñeca y dedos, incrementando el control de tronco en dos puntos con apoyo (mayo 2003).

observados en miembro superior fueron más claros que los de miembro inferior en este caso. Esto confirma los beneficios reportados por Aguilar-Rebolledo y col. en el 2001, quienes observaron mayor eficiencia de la TBA en la espasticidad asociada a distonía en el miembro superior. Cabe recordar que posterior a la aplicación de la TBA, se inició un programa de fisioterapia organizado para mejorar el AMP, en cuanto a la coordinación gruesa y fina, ya que los músculos habían sufrido acortamiento y pérdida funcional, especialmente la musculatura isquiotibial, con una contractura dinámica.

El efecto esperado (relajación o disminución de la espasticidad) varía considerablemente para una misma dosis en el mismo músculo de diferentes pacientes. Esto parece estar dado por el grado y el tiempo de evolución de la espasticidad, las medidas de rehabilitación previas; o quizá pueda ser debida a un efecto técnico relacionado con la infiltración (su cercanía o alejamiento con las placas neuromotoras), lo cual puede condicionar la intensidad del efecto. La contractura dinámica de los músculos isquiotibiales en este caso fue el gran obstáculo para producir un efecto de mayor intensidad de la TBA.

Frecuentemente los niños con PCI prácticamente no utilizan los músculos antagonistas de los músculos espásticos, de tal suerte que, al permitir la relajación de estos músculos mediante la TBA, no es suficiente para activarlos y esto se consigue a través de la rehabilitación. El efecto de la fisioterapia y de la TBA es sinérgico, además de los apoyos ortésicos. Con el resultado obtenido en este caso y los encontrados en la literatura $(1,2,4,6-9,11-16)$, podemos confirmar que este tratamiento es adecuado para el manejo de la espasticidad en la PCI. Los efectos benéficos prdrían prolongarse y lograrse más fácilmente, si la aplicación de la TBA se asocia con un adecuado programa de fisioterapia individualizado, medidas ortésicas y la potencial asociación con cirugía ortopédica.

Asimismo, la ganancia funcional potencial debe preverse en un consenso multidisciplinario. Uno de los conceptos más importantes es que el tratamiento con TBA debe llevarse a cabo por un profesional con experiencia, que conozca con claridad la neuroanatomía y la función de cada uno de los músculos que se requieren tratar.

\section{AGRADECIMIENTOS.}

Le agradezco al MC. Francisco Aguilar-Rebolledo y al MC. Russell René Arcila-Novelo por su colaboración en la técnica de aplicación de la inyección de Toxina Botulínica A. Le agradezco al laboratorio Allergan por la donación del BOTOX type A para su utilización en este caso.

\section{REFERENCIAS.}

1. Roslyn B, Grahan HK. Botulinum toxin A in the management of children with cerebral palsy: indications and outcomes. Eur J Neurol 1997; 4 (Suppl 2):15-22.

2. Davis EC, Barnes MP. Botulinum toxin and spasticity. J Neurol Neurosurg Psych 2000; 69: 143-7.

3. Singer C. Indicaciones y manejo de la toxina botulínica. Rev Ecua Neurol 2000; 9 (1 y 2). En http:// www.medicosecuador.com/revecuatneurol/Vol9 n1-2 2000. html

4. Herrera-Galante A, Pérez-Fernández F, Flores-Jurado M, Gómez-González A, Godoy-Ramírez AM. Tratamiento de la espasticidad con toxina botulínica en la parálisis cerebral infantil. Rehabilitación 1999; 33: 304 - 9

5. Arnold SS, Schechter R, Inglesby TV, Henderson DA, Bartlett JG, Asher MS, et al. Botulinum toxin as a biological weapon. JAMA 2001; 285: 1059-70. 
112

\section{A Ojeda-Manzano.}

6. Aguilar-Rebolledo F, Hernández-Sanchez J, Rayo-Mares $\mathrm{D}$, Soriano-Fonseca F, García-Muñoz L, Ruiz-Ponce J, et al. Toxina Botulínica como tratamiento de la espasticidad y distonía de la parálisis cerebral infantil. Gac Méd Méx 2001; 137:403-11.

7. Cosgrove AP, Corry IS, Graham HK. Botulinum toxin in the management of the lower limb in cerebral palsy. Dev Med Child Neurol 1994; 36: 386-96.

8. Churotian AM, Root L. Management of spasticity in children with Botulinum-A toxin. Int Pediatr 1994; 9: 129-37.

9. Gormley ME, Herring GM, Gaebler-Spira D. The use of botulinum toxin in children: a retrospective study of adverse reactions and treatment of idiophatic toe-walking. Eur $\mathbf{J}$ Neurol 1997; 4 (Suppl 2): 27-30.

10. Aguilar-Rebolledo F. Pascual-Pascual S.I. Tratamientos farmacológicos para la parálisis cerebral. I Tratamiento de la Espasticidad. Plasticidad y Restauración neurológica. Plast $\&$ rest neurol 2002; 1:23-32.

11. Dune JW, Heye N, Dunne SL. Treatment for chronic limb spasticity with botulinum toxin A. J Neurol Neurosurg Psychiatriy 1995; 58:232-5.

12. Chutorian A, Root L, BTA Study Group. A multicenter randomized, double-mind, placebo controlled trial of Botulinum toxin type A (BTA) in the treatment of lower limb spasticity in pediatric cerebral palsy. Mov Disord 1995; 10:364.

13. Simpson DM, Alexander DN, O'Brien CF, Tagliati M, Aswad AS, Leon JM, et al. Botulinum toxin type A in the treatment of upper extremity. A randomized, double-blind, placebo-controlled trial. Neurology 1996; 46:1306-10.

14. Pullman SL, Greene P, Fahn S, Pedersen SF. Approach to the treatment of limb disorders with Botulinum toxin A. Experience with 187 patients. Arch Neurol 1996; 53:617-24.

15. Grazko MA, Polo KB, Jabbari B. Botulinum toxin A for spasticity, muscle spasms, and rigidity. Neurology 1995; 45: 712-7.

16. Ruiz-Martínez BA, Salas-Roldán E. Toxina botulínica y fisioterapia en la parálisis cerebral infantil. Fisioterapia 2002; 24:128-31.

\section{Revista Biomédica}

